Homology, Homotopy and Applications, vol.21(1), 2019, pp.269-281

\title{
LINEARITY PROBLEM FOR NON-ABELIAN TENSOR PRODUCTS
}

\author{
VALERIY G. BARDAKOV, ANDREI V. LAVRENOV AND \\ MIKHAIL V. NESHCHADIM \\ (communicated by Ronald Brown)
}

\begin{abstract}
In this paper we give an example of a linear group such that its tensor square is not linear. Also, we formulate some sufficient conditions for the linearity of non-abelian tensor products $G \otimes$ $H$ and tensor squares $G \otimes G$. Using these results we prove that tensor squares of some groups with one relation and some knot groups are linear. We prove that the Peiffer square of a finitely generated linear group is linear. At the end we construct faithful linear representations for the non-abelian tensor square of a free group and free nilpotent group.
\end{abstract}

\section{Introduction}

Brown and Loday $[6,7]$ introduced the non-abelian tensor product $G \otimes H$ for a pair of groups $G$ and $H$ following works of Miller [16], and Lue [14]. They showed that the third homotopy group of the suspension of an Eilenberg-MacLane space $K(G, 1)$ satisfies

$$
\pi_{3} S K(G, 1) \cong J_{2}(G),
$$

where $J_{2}(G)$ is the kernel of the derived map $\kappa: G \otimes G \longrightarrow G^{\prime}, g \otimes h \mapsto[g, h]=$ $g^{-1} h^{-1} g h$. Hence there exists the short exact sequence

$$
0 \longrightarrow \pi_{3} S K(G, 1) \longrightarrow G \otimes G \longrightarrow G^{\prime} \longrightarrow 1 \text {. }
$$

Also, the non-abelian tensor product is used to compute some homotopy 3-types [4] and to describe the third relative homotopy group of a triad as a non-abelian tensor product of the second homotopy groups of appropriate subspaces. More specifically, let a $C W$-complex $X$ be the union $X=A \cup B$ of two pointed path-connected $C W$ subspaces $A$ and $B$ whose intersection $C=A \cap B$ is path-connected. If the canonical homomorphisms $\pi_{1}(C) \longrightarrow \pi_{1}(A), \pi_{1}(C) \longrightarrow \pi_{1}(B)$ are surjective, then, according to $[6]$,

$$
\pi_{3}(X, A, B) \cong \pi_{2}(A, C) \otimes \pi_{2}(B, C),
$$

where the groups $\pi_{2}(A, C)$ and $\pi_{2}(B, C)$ act on one another via $\pi_{1}(C)$.

Received April 11, 2018, revised August 16, 2018; published on October 17, 2018.

2010 Mathematics Subject Classification: 20E25, 20G20, 20 E05.

Key words and phrases: non-abelian tensor product, linear group, faithful linear representation.

Article available at http://dx.doi.org/10.4310/HHA.2019.v21.n1.a12

Copyright (C) 2018, International Press. Permission to copy for private use granted. 
The investigation of the non-abelian tensor product from a group theoretical point of view started with a paper by Brown, Johnson, and Robertson [5]. They compute the non-abelian tensor square of all non-abelian groups of order up to 30 using Tietze transformations.

One of the topics of research on the non-abelian tensor products has been to determine which group properties are preserved by non-abelian tensor products. By using homological arguments, Ellis [10] showed that if $G$ and $H$ are finite groups, then $G \otimes H$ is also finite. Visscher [20] proved that if $G, H$ are solvable (nilpotent), then $G \otimes H$ is solvable (nilpotent) and gives a bound on the nilpotency class of $G \otimes H$. In [9] it was proved that the tensor product of groups of nilpotency class at most $n$ is a group of nilpotency class at most $n$, thereby improving the bound given by Visscher. For other results in this direction see the survey of Nakaoka [18].

In this paper we study the linearity problem for non-abelian tensor products. Let $n$ be a positive integer and let $P$ be a field. A group $G$ is said to be linear of degree $n$ over $P$ if it is isomorphic with a subgroup of $G L_{n}(P)$, the group of all $n \times n$ nonsingular matrices over $P$ or, equivalently, if it is isomorphic with a group of invertible linear transformations of a vector space of dimension $n$ over $P$ (see [15]). We study the following question.

Let $G$ and $H$ be linear groups. Are the groups $G \otimes H, G \otimes G$ linear?

We show that in general the answer is negative. More accurately, we prove that the tensor square $S L_{n}(\mathbb{Q}) \otimes S L_{n}(\mathbb{Q})$ of the special linear group $S L_{n}(\mathbb{Q})$ over the field of rational numbers is not linear for $n \geqslant 3$. On the other side we formulate some sufficient conditions under which the groups $G \otimes H, G \otimes G$ are linear. Using these conditions, we prove that the non-abelian tensor squares of some groups with one defining relation and groups of fibered knots are linear. If $G$ is a finitely generated free group or finitely generated free nilpotent group, then we construct concrete faithful linear representations for $G \otimes G$.

The non-abelian tensor square $G \otimes G$ is connected to other group constructions: exterior tensor square $G \wedge G$ and Peiffer square $G \bowtie G$. We prove that if $G$ is finitely generated, then $G \bowtie G$ is linear.

We note that the following problems are still open:

1) Let $G$ be a finitely generated linear group. Is the group $G \otimes G$ linear?

2) Let $G$ be a linear group. Is the group $G \bowtie G$ linear?

\section{Acknowledgments}

The authors are grateful to V. Thomas, S.O. Ivanov, V. Petrushenko, A.Yu. Ol'shanskii, V.A. Roman'kov, M. Singh for useful discussions. Also we thank to J.O. Button and O.V. Bryukhanov for examples of linear groups with non-linear abelianization (see Section 5). The present work was supported by Russian Science Foundation (project No. 16-41-02006).

\section{Preliminaries}

In this paper we shall use the following notations. For elements $x, y$ in a group $G$, the conjugation of $x$ by $y$ is $x^{y}=y^{-1} x y$; and the commutator of $x$ and $y$ is 
$[x, y]=x^{-1} x^{y}=x^{-1} y^{-1} x y$. We write $G^{\prime}$ for the derived subgroup of $G, G^{a b}$ for the abelianized group $G / G^{\prime}$.

Recall the definition of the non-abelian tensor product $G \otimes H$ of groups $G$ and $H$ (see $[6,7]$ ). This tensor product is defined for any pair of groups $G$ and $H$ where each one acts on the other (on the right)

$$
G \times H \longrightarrow G, \quad(g, h) \mapsto g^{h} ; \quad H \times G \longrightarrow H, \quad(h, g) \mapsto h^{g}
$$

and on itself by conjugation, in such a way that for all $g, g_{1} \in G$ and $h, h_{1} \in H$,

$$
g^{\left(h^{g_{1}}\right)}=\left(\left(g^{g_{1}^{-1}}\right)^{h}\right)^{g_{1}} \quad \text { and } \quad h^{\left(g^{h_{1}}\right)}=\left(\left(h^{h_{1}^{-1}}\right)^{g}\right)^{h_{1}} .
$$

In this situation we say that $G$ and $H$ act compatibly on each other. The non-abelian tensor product $G \otimes H$ is the group generated by all symbols $g \otimes h, g \in G, h \in H$, subject to the relations

$$
g g_{1} \otimes h=\left(g^{g_{1}} \otimes h^{g_{1}}\right)\left(g_{1} \otimes h\right) \quad \text { and } \quad g \otimes h h_{1}=\left(g \otimes h_{1}\right)\left(g^{h_{1}} \otimes h^{h_{1}}\right)
$$

for all $g, g_{1} \in G, h, h_{1} \in H$.

In particular, as the conjugation action of a group $G$ on itself is compatible, then the tensor square $G \otimes G$ of a group $G$ may always be defined. Also, the tensor product $G \otimes H$ is defined if $G$ and $H$ are two normal subgroups of some group $M$ and actions are conjugations in $M$.

Recall the main diagram for the non-abelian tensor square (see [6, 7]). Let $G$ be a group. One of the main tools for studying of the non-abelian tensor square $G \otimes G$ is the following diagram:

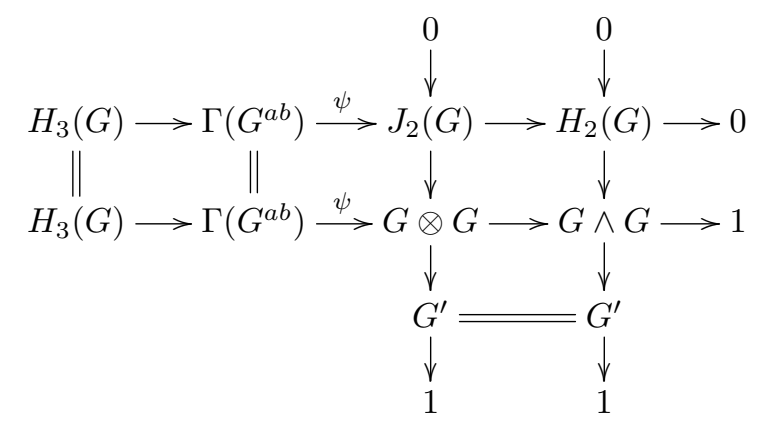

with exact rows and columns. Here:

1) $H_{2}(G), H_{3}(G)$ are the second and the third homology groups of $G$ with the coefficients in the trivial $\mathbb{Z} G$-module $\mathbb{Z}$. The second homology group $H_{2}(G)$ for the group $G=F / R$, where $F$ is a free group, can be found by the Hopf formula:

$$
H_{2}(G) \cong\left(F^{\prime} \cap R\right) /[F, R]
$$

2) $G \wedge G$ is the exterior product of $G$ onto itself. For the group $G=F / R$ it can be presented in the form (see [2])

$$
G \wedge G \cong F^{\prime} /[F, R]
$$

In particular, if $G$ is a free group, then $G \wedge G \cong G^{\prime}$. 
3) $J_{2}(G)=\pi_{3} S K(G, 1)$ is the kernel of the derived map $\kappa: G \otimes G \longrightarrow G^{\prime}$, which on the generators of $G \otimes G$ is defined by the rule:

$$
g_{1} \otimes g_{2} \mapsto\left[g_{1}, g_{2}\right] .
$$

The group $J_{2}(G)$ lies in the center $Z(G \otimes G)$ and its elements are invariant under the action of $G$ onto $G \otimes G$, which is defined by the formula

$$
\left(g_{1} \otimes g_{2}\right)^{g}=g_{1}^{g} \otimes g_{2}^{g} .
$$

In particular, if $g_{2}=g_{1}$, then

$$
\left(g_{1} \otimes g_{1}\right)^{g}=g_{1} \otimes g_{1}
$$

for any $g, g_{1} \in G$.

4) $\Gamma\left(G^{a b}\right)$ is Whitehead's quadratic functor. The group $\Gamma\left(G^{a b}\right)$ is generated by elements $\gamma\left(g G^{\prime}\right)$ and $\psi$ is defined by the formula

$$
\gamma\left(g G^{\prime}\right) \longmapsto g \otimes g .
$$

The image $\psi \Gamma\left(G^{a b}\right)$ is not equal in the general case to the group $J_{2}(G)$ since $J_{2}(G) / \psi \Gamma\left(G^{a b}\right) \cong H_{2}(G)$.

For the functor $\Gamma: A \longmapsto \Gamma(A)$, where $A$ is an abelian group it is known that:

a) $\Gamma(A \times B) \cong \Gamma(A) \times \Gamma(B) \times\left(A \otimes_{\mathbb{Z}} B\right)$, where $A \otimes_{\mathbb{Z}} B$ is the abelian tensor product of abelian groups.

b) $\Gamma\left(\mathbb{Z}_{n}\right) \cong \begin{cases}\mathbb{Z}_{n} & n \equiv 1(\bmod 2), \\ \mathbb{Z}_{2 n} & n \equiv 0(\bmod 2) .\end{cases}$

c) $\Gamma(\mathbb{Z}) \cong \mathbb{Z}$.

In particular, $\Gamma\left(\mathbb{Z}^{n}\right) \cong \mathbb{Z}^{\frac{n(n+1)}{2}}$.

\section{Linearity problem}

In this section we will use a result of Malcev [15] (see also [21, Chapter 2]) on the linearity of abelian groups. To formulate it, recall some definitions. If $G$ is any group $\tau(G)$ is the subgroup of $G$ generated by all the periodic normal subgroups of $G$; that is $\tau(G)$ is the maximum periodic normal subgroup of $G$. $G$ has finite rank at most $n$ if every finite subset of $G$ is contained in an $n$-generator subgroup of $G$. If $G$ is abelian and periodic then $G$ has finite rank at most $n$ if and only if for each prime $p$ the Sylow $p$-subgroup of $G$ is a direct product of at most $n$ cyclic and $\mathbb{C}_{p^{\infty}}$-groups. If $\pi$ is any set of primes and $G$ is a group with a unique maximal $\pi$-subgroup we denote this maximal $\pi$-subgroup by $G_{\pi}$.

Malcev proved:

i) An abelian group $A$ has a faithful representation of degree $n \geqslant 1$ over some field of characteristic zero if and only if $\tau(G)$ has rank at most $n$.

ii) An abelian group $A$ has a faithful representation of degree $n \geqslant 1$ over some field of characteristic $p>0$ if and only if $\tau(G)_{p^{\prime}}$ (here $p^{\prime}$ denotes all primes except $p$ ) has finite rank $r$ and $\tau(A)_{p}$ has finite exponent $p^{e}$ satisfying

$$
p^{e-1}+\max \{1, r\}<n+1 .
$$

We are ready to prove the following 
Proposition 3.1. There is a linear group $G$ such that $G \otimes G=G \wedge G$ is not linear.

Proof. For a perfect group $G=G^{\prime}$ it follows from the main diagram (see Section 2) that $G \otimes G=G \wedge G$ and the sequence

$$
0 \longrightarrow H_{2}(G, \mathbb{Z}) \longrightarrow G \otimes G \longrightarrow G \longrightarrow 0
$$

is exact.

For $n \geqslant 3$ the group $S L_{n}(\mathbb{Q})$ is perfect and its second homology group coincides with the $\mathrm{K}_{2}$-group of the field $\mathbb{Q}$,

$$
H_{2}\left(S L_{n}(\mathbb{Q}), \mathbb{Z}\right)=\mathrm{K}_{2}(\mathbb{Q}),
$$

see [17, Corollary 11.2].

Next,

$$
\mathrm{K}_{2}(\mathbb{Q})=\{ \pm 1\} \times \prod_{p \text { odd prime }}(\mathbb{Z} / p)^{\times}
$$

by $[17$, Theorem 11.6$]$, so that $K_{2}(\mathbb{Q})$ contains an abelian 2-group of infinite rank and unbounded exponent. Using Malcev's criterion we conclude that such a group can not be linear. Therefore the group

$$
S L_{n}(\mathbb{Q}) \otimes S L_{n}(\mathbb{Q})=S L_{n}(\mathbb{Q}) \wedge S L_{n}(\mathbb{Q})
$$

is not linear as well.

To study the linearity problem for the non-abelian tensor product we can use a presentation of a tensor product as a central extension (see, for example, [9]). The derivative subgroup of $G$ by $H$ is defined to be the following subgroup

$$
D_{H}(G)=[G, H]=\left\langle g^{-1} g^{h} \mid g \in G, h \in H\right\rangle .
$$

The map $\kappa: G \otimes H \longrightarrow D_{H}(G)$ defined by $\kappa(g \otimes h)=g^{-1} g^{h}$ is a homomorphism, its kernel is the central subgroup of $G \otimes H$ and $G$ acts on $G \otimes H$ by the rule $(g \otimes h)^{x}=$ $g^{x} \otimes h^{x}, x \in G$. There exists the short exact sequence

$$
1 \longrightarrow A \longrightarrow G \otimes H \longrightarrow D_{H}(G) \longrightarrow 1 \text {. }
$$

In this case, $A$ can be viewed as a $\mathbb{Z}\left[D_{H}(G)\right]$-module via conjugation in $G \otimes H$, i.e. under the action induced by setting

$$
a \cdot g=x^{-1} a x, \quad a \in A, x \in G \otimes H, \kappa(x)=g .
$$

We can formulate some sufficient conditions for the linearity of $G \otimes H$. It is well known that the tensor product $G \otimes H$ with trivial actions is isomorphic to the abelian tensor product $G^{a b} \otimes_{\mathbb{Z}} H^{a b}$. Hence, in this case the question on the linearity of $G \otimes H$ is equivalent to the question on the linearity of the abelian tensor product and the answer follows from the Malcev theorem.

Further we will assume that the action of $G$ on $H$ or the action of $H$ on $G$ is non-trivial. We have the following short exact sequence

$$
0 \longrightarrow A \longrightarrow G \otimes H \longrightarrow D_{H}(G) \longrightarrow 1 \text {. }
$$

Note that $A$ is the kernel of the natural map $G \otimes H \longrightarrow D_{H}(G), g \otimes h \longrightarrow g^{-1} g^{h}$, $g \in G, h \in H$, and is a central subgroup of $G \otimes H$. 
Proposition 3.2. Let the following conditions hold:

1) $A, D_{H}(G)$ are linear groups.

2) $H^{2}\left(D_{H}(G), A\right)=0$, in particular, this condition holds if $A$ is divisible or $D_{H}(G)$ is a free group.

Then $G \otimes H=A \times D_{H}(G)$ is a linear group.

Proof. It is well known (see, for example [3, Chapter IV]) that if $H^{2}\left(D_{H}(G), A\right)=0$, then the sequence

$$
0 \longrightarrow A \longrightarrow G \otimes H \longrightarrow D_{H}(G) \longrightarrow 1
$$

splits. In particular, this condition holds if $A$ is divisible or $D_{H}(G)$ is a free group.

Since $A$ is a central subgroup, then $G \otimes H \cong A \times D_{H}(G)$ and is a linear group as a direct product of linear groups.

The main problem in the use of this theorem is the description of the central subgroup $A$. For the tensor square we can use another approach.

Let us formulate some sufficient conditions under which $G \otimes G$ is a direct product of the commutator subgroup $G^{\prime}$ and the Whitehead group $\Gamma\left(G^{a b}\right)$.

Theorem 3.3. Let $H_{2}(G)=H_{3}(G)=H_{2}\left(G^{\prime}\right)=0$ and one of the following conditions hold:

1) $H^{2}\left(G^{\prime}, \Gamma\left(G^{a b}\right)\right)=0$;

2) $\Gamma\left(G^{a b}\right)$ is a divisible group;

3) $G^{\prime} / G^{\prime \prime}$ is a free abelian group.

Then

$$
G \otimes G \cong \Gamma\left(G^{a b}\right) \times G^{\prime} .
$$

If, moreover, $G$ is finitely generated and $G^{\prime}$ is linear, then $G \otimes G$ is linear.

Proof. Since, $H_{2}(G)=H_{3}(G)=0$, then the main diagram has the form

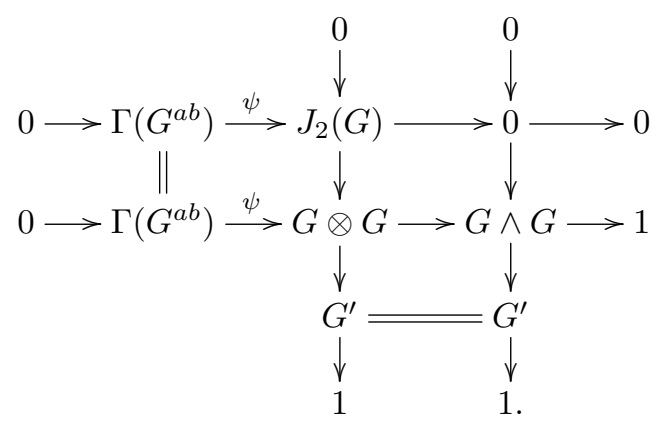

From this diagram $J_{2}(G)=\Gamma\left(G^{a b}\right)$ and $G \wedge G=G^{\prime}$. Hence we have the short exact sequence

$$
0 \longrightarrow \Gamma\left(G^{a b}\right) \longrightarrow G \otimes G \longrightarrow G^{\prime} \longrightarrow 1 .
$$

If $H^{2}\left(G^{\prime}, \Gamma\left(G^{a b}\right)\right)=0$, then this sequence is splittable:

$$
G \otimes G=\Gamma\left(G^{a b}\right) 入 G^{\prime} .
$$

As we know that $\Gamma\left(G^{a b}\right)$ is divisible or $G^{\prime}$ is free, then $H^{2}\left(G^{\prime}, \Gamma\left(G^{a b}\right)\right)=0$. Let us show that if $G^{\prime} / G^{\prime \prime}$ does not have torsion, then $H^{2}\left(G^{\prime}, \Gamma\left(G^{a b}\right)\right)=0$. Indeed, by the 
universal coefficient theorem there is the following short exact sequence

$$
0 \longrightarrow \operatorname{Ext}_{\mathbb{Z}}\left(H_{1}\left(G^{\prime}\right), \Gamma\left(G^{a b}\right)\right) \longrightarrow H^{2}\left(G^{\prime}, \Gamma\left(G^{a b}\right)\right) \longrightarrow \operatorname{Hom}_{\mathbb{Z}}\left(H_{2}\left(G^{\prime}\right), \Gamma\left(G^{a b}\right)\right) \longrightarrow 0 .
$$

Since $H_{2}\left(G^{\prime}\right)=0$ we have the short exact sequence

$$
0 \longrightarrow \operatorname{Ext}_{\mathbb{Z}}\left(H_{1}\left(G^{\prime}\right), \Gamma\left(G^{a b}\right)\right) \longrightarrow H^{2}\left(G^{\prime}, \Gamma\left(G^{a b}\right)\right) \longrightarrow 0 .
$$

Hence $H^{2}\left(G^{\prime}, \Gamma\left(G^{a b}\right)\right)=0$ if and only if $\operatorname{Ext}_{\mathbb{Z}}\left(H_{1}\left(G^{\prime}\right), \Gamma\left(G^{a b}\right)\right)=0$. It is known that if $H_{1}\left(G^{\prime}\right)$ is free abelian, then $\operatorname{Ext}_{\mathbb{Z}}\left(H_{1}\left(G^{\prime}\right), \Gamma\left(G^{a b}\right)\right)=0$.

Since $\Gamma\left(G^{a b}\right)$ is a central subgroup, this product is the direct product:

$$
G \otimes G=\Gamma\left(G^{a b}\right) \times G^{\prime} .
$$

If $G$ is a finitely generated, then $G^{a b}$ is finitely generated abelian group and $\Gamma\left(G^{a b}\right)$ is also a finitely generated abelian group. Then $G \otimes G$ is linear as a direct product of two linear groups.

As consequence we get the following result:

Corollary 3.4. [2] Let $F_{n}$ be a free group of rank $n$. Then

$$
F_{n} \otimes F_{n} \cong \mathbb{Z}^{n(n+1) / 2} \times\left(F_{n}\right)^{\prime} .
$$

\section{Groups with one defining relation and knot groups}

Let $G$ be a group with one defining relation:

$$
G=\langle X \| r=1\rangle,
$$

where $r \notin F^{\prime}, F=\langle X\rangle$. Then $H_{k}(G)=0, k \geqslant 2$ (see [3, p. 49]). Hence, there exists the following short exact sequence:

$$
0 \longrightarrow \Gamma\left(G^{a b}\right) \longrightarrow G \otimes G \longrightarrow G^{\prime} \longrightarrow 1
$$

If $G^{a b}$ does not have torsion, then $G^{a b}$ is a free abelian group and $\Gamma\left(G^{a b}\right)$ is a free abelian group. Then, if $H^{2}\left(G^{\prime}\right)=0$, then $H^{2}\left(G^{\prime}, \Gamma\left(G^{a b}\right)\right)=0$, which follows from the decomposition

$$
H^{k}(S, A \oplus B)=H^{k}(S, A) \oplus H^{k}(S, B)
$$

for every group $S$ and all $S$-modules $A$ and $B$.

From Theorem 3.3 follows

Proposition 4.1. Let $G$ be a group with one defining relation:

$$
G=\langle X \| r=1\rangle,
$$

where $r \notin F^{\prime}, F=\langle X\rangle$ such that $H^{2}\left(G^{\prime}\right)=0$. If one from the following conditions holds:

1) $G^{a b}$ does not have torsion,

2) $G^{\prime} / G^{\prime \prime}$ is a free abelian group,

then $G \otimes G=\Gamma\left(G^{a b}\right) \times G^{\prime}$. If, moreover, $G$ is finitely generated and $G$ is linear, then $G \otimes G$ is linear. 
It is well known that if $K$ is a tame knot in 3 -sphere $\mathbb{S}^{3}$ and $G_{K}=\pi_{1}\left(\mathbb{S}^{3} \backslash K\right)$ its group, then $H_{n}\left(G_{K}\right)=0$ for $n>1$ (see, for example [13, p. 5]). Recall that a knot $K$ is called fibered if there is a 1-parameter family $F_{t}$ of Seifert surfaces for $K$, where the parameter $t$ runs through the points of the unit circle $S^{1}$, such that if $s$ is not equal to $t$ then the intersection of $F_{s}$ and $F_{t}$ is exactly $K$. The commutator subgroup $G_{K}^{\prime}$ for the fibered knot $K$ is a free group of finite rank [8] and $G_{K}$ is linear [1].

Proposition 4.2. Let $K$ be a tame fibered knot in 3-sphere $\mathbb{S}^{3}$, then $G_{K} \otimes G_{K}=$ $G_{K}^{\prime} \times \mathbb{Z}$ and has a faithful linear representation into $G L_{2}\left(\mathbb{Z}\left[t, t^{-1}\right]\right)$.

Proof. It is well known that $G_{K}^{a b}=\mathbb{Z}$ and then $\Gamma\left(G_{K}^{a b}\right)=\mathbb{Z}$. From Theorem 3.3 it follows that $G_{K} \otimes G_{K}=\mathbb{Z} \times G_{K}^{\prime}$.

To construct a linear representation, use the fact that $G_{K}^{\prime}$ is a free group of finite rank and by Sanov's theorem [12, Chapter 5] it has a faithful linear representation into $S L_{2}(\mathbb{Z}) \leqslant G L_{2}\left(\mathbb{Z}\left[t, t^{-1}\right]\right)$. Define a linear representation of $G_{K}^{a b}=\mathbb{Z}=\langle\gamma\rangle$ into $G L_{2}\left(\mathbb{Z}\left[t, t^{-1}\right]\right)$ by the rule

$$
\gamma \longmapsto\left(\begin{array}{cc}
t & 0 \\
0 & t
\end{array}\right)
$$

Since the image of $\gamma$ is a scalar matrix, i.e. lies in the center of $G L_{2}\left(\mathbb{Z}\left[t, t^{-1}\right]\right)$, we constructed a faithful linear representation of $G_{K} \otimes G_{K}$.

Example 4.3. 1) The braid group $B_{3}$ on 3 strings has presentation

$$
B_{3}=\left\langle\sigma_{1}, \sigma_{2} \| \sigma_{1} \sigma_{2} \sigma_{1}=\sigma_{2} \sigma_{1} \sigma_{2}\right\rangle
$$

and is the group of trefoil knot. The commutator subgroup $B_{3}^{\prime}$ is a free group of rank 2 . Hence the tensor square $B_{3} \otimes B_{3}=\mathbb{Z} \times F_{2}$ has a faithful linear representation into $G L_{2}\left(\mathbb{Z}\left[t, t^{-1}\right]\right)$.

2) It is known that the group of the figure eight knot has a presentation

$$
G=\left\langle x, y \| y x^{-1} y x y^{-1}=x^{-1} y x y^{-1} x\right\rangle
$$

and is a fibered knot. Hence the tensor square $G \otimes G=\mathbb{Z} \times G^{\prime}$ has a faithful linear representation into $G L_{2}\left(\mathbb{Z}\left[t, t^{-1}\right]\right)$.

In the first example we showed that $B_{3} \otimes B_{3}=\mathbb{Z} \times F_{2}$. On the other side $B_{3}$ contains the pure braid group $P_{3}$, which is normal in $B_{3}$, has index 6 and is the direct product of the center, which is isomorphic to $\mathbb{Z}$, and a free group of rank 2 . Hence, $B_{3} \otimes B_{3}$ is isomorphic to $P_{3}$ and we proved

Proposition 4.4. There is a non-trivial non-abelian group $G$ such that the tensor square $G \otimes G$ is isomorphic to a proper subgroup of $G$.

We note that the following problems are still open:

1) Is it true that $B_{n} \otimes B_{n}, n>3$, is linear?

2) Is it true that for arbitrary tame knot $K$ the group $G(K) \otimes G(K)$ is linear?

\section{On the linearity of the Peiffer product}

Recall the definition of the Peiffer product. Given $G$ and $H$ acting compatibly on each other, in [22] the Peiffer product $G \bowtie H$ was defined their as the quotient of the 
free product $G * H$ by the normal closure $K$ of all elements of the form

$$
h^{-1} g^{-1} h g^{h} \quad \text { or } \quad g^{-1} h^{-1} g h^{g},
$$

where $g \in G$ and $h \in H$. Whitehead [22] posed a question on the asphericity of subcomplexes of aspherical 2-complexes and reformulated it as part of the wider problem of finding conditions under which the groups $G$ and $H$ are embedded in $G \bowtie H$.

In [11] it was proved that if $\varphi: G * H \rightarrow G \bowtie H$, then modulo $K=\operatorname{Ker}(\varphi), h g \equiv$ $g h^{g}$, so that every element of $G \bowtie H$ can be written as $\varphi(g) \varphi(h)$ for suitable $g, h$. Denote $\varphi(g) \varphi(h)$ as $\langle g, h\rangle$. The relations

$$
\langle g, h\rangle\left\langle g_{1}, h_{1}\right\rangle=\left\langle g g_{1}, h^{g_{1}} h_{1}\right\rangle=\left\langle g g_{1}^{h^{-1}}, h h_{1}\right\rangle
$$

are defining relations for $G \bowtie H$ on the generators $\langle g, h\rangle$ and so $G \bowtie H$ is a homomorphic image of both the semidirect products $G \ltimes H$ and $G \rtimes H$. The group $G \bowtie H$ is obtained from $G \ltimes H$ (or from $G \rtimes H$ ) by imposing the relations

$$
\left(g^{-1} g^{h}, 1\right)=\left(1, h^{-g} h\right) \text {. }
$$

If $G$ and $H$ act on one another trivially, then $G \bowtie H$ is just the direct product $G \times H$ and $K=G \square H$, where $G \square H$ is the Cartesian subgroup of $G * H$ (the kernel of the canonical homomorphism $G * H \longrightarrow G \times H)$.

From [11, Proposition 2.1] follows

$$
G \bowtie G \cong G^{a b} \times G^{a b} .
$$

Using this isomorphism one can prove

Proposition 5.1. Let $G$ be a linear group and $G$ is finitely generated or $G=G^{\prime}$, then $G \bowtie G$ is linear.

From this proposition it follows that if $G=S L_{n}(\mathbb{Q}), n \geqslant 2$, then $G \bowtie G$ is linear. On the other side, we know that $S L_{n}(\mathbb{Q}) \otimes S L_{n}(\mathbb{Q})$ and $S L_{n}(\mathbb{Q}) \wedge S L_{n}(\mathbb{Q})$ are not linear for $n \geqslant 3$.

Note that this proposition is not true for arbitrary linear group $G$ since there are linear groups with nonlinear abelianization.

Example 5.2. 1) (O.V. Bryukhanov) Let $G=\underset{i=2}{*} \underset{Z}{\infty}$ be the free product of cyclic groups. Then $G$ is linear as the free product of linear groups. On the other side, by Malcev criteria (see Section 3 ) the abelianization $G^{a b}$ is not linear.

2) (J.O. Button) Take the set of matrices

$$
A_{i}=\left(\begin{array}{cc}
1 & x^{i} \\
0 & 1
\end{array}\right) \in S L_{2}(\mathbb{Z}[x]), \quad i \in \mathbb{N} .
$$

Then $A=\left\langle A_{i} \mid i \in \mathbb{N}\right\rangle$ is a free abelian group of countable rank. Put

$$
B=\left(\begin{array}{ll}
3 & 0 \\
0 & 1
\end{array}\right) \in G L_{2}(\mathbb{Q}) .
$$

It is easily to check that these matrices satisfy the relations

$$
B A_{i} B^{-1}=A_{i}^{3}, \quad i \in \mathbb{N} .
$$


Hence the group generated by $A_{i}$ and $B$ has the presentation

$$
G_{2}=\left\langle A_{i}, i \in \mathbb{N}, B \|\left[A_{i}, A_{j}\right]=1, B A_{i} B^{-1}=A_{i}^{3}, i, j \in \mathbb{N}\right\rangle
$$

which is a subgroup of $G L_{2}(\mathbb{Q}[x])$, but its abelianization $G_{2}^{a b} \cong \bigoplus_{i=1}^{\infty} \mathbb{Z}_{2} \oplus \mathbb{Z}$ does not have a faithful linear representations over field of characteristic $p \neq 2$.

Analogously, take the set of matrices

$$
C_{i}=\left(\begin{array}{cc}
1 & y^{i} \\
0 & 1
\end{array}\right) \in S L_{2}(\mathbb{Z}[y]), \quad i \in \mathbb{N} .
$$

Then $C=\left\langle C_{i} \mid i \in \mathbb{N}\right\rangle$ is a free abelian group of countable rank. Put

$$
D=\left(\begin{array}{ll}
4 & 0 \\
0 & 1
\end{array}\right) \in G L_{2}(\mathbb{Q}) .
$$

It is easily to check that these matrices satisfy the relations

$$
D C_{i} D^{-1}=C_{i}^{4}, \quad i \in \mathbb{N} .
$$

Hence the group generated by $C_{i}$ and $D$ has the presentation

$$
G_{3}=\left\langle C_{i}, i \in \mathbb{N}, D \|\left[C_{i}, C_{j}\right]=1, D C_{i} D^{-1}=C_{i}^{4}, i, j \in \mathbb{N}\right\rangle,
$$

which is a subgroup of $G L_{2}(\mathbb{Q}[y])$, but its abelianization $G_{3}^{a b} \cong \bigoplus_{i=1}^{\infty} \mathbb{Z}_{3} \oplus \mathbb{Z}$ does not have a faithful linear representations over field of characteristic $p \neq 3$.

Let us take $G=G_{2} \oplus G_{3}$. Then $G$ is metabelian and has a faithful linear representation in $G L_{4}(\mathbb{Q}[x, y])$, but its abelianization $G^{a b} \cong \bigoplus_{i=1}^{\infty}\left(\mathbb{Z}_{2} \oplus \mathbb{Z}_{3}\right) \oplus \mathbb{Z} \oplus \mathbb{Z}$ is not linear.

It is evident that the sequence

$$
1 \longrightarrow 1 \times G^{\prime} \longrightarrow G^{a b} \times G \longrightarrow G^{a b} \times G^{a b} \longrightarrow 1
$$

is short exact. Since $G^{a b} \times G \cong G \bowtie G$ we can add the following new terms into the main diagram.

Proposition 5.3. The following diagram holds:

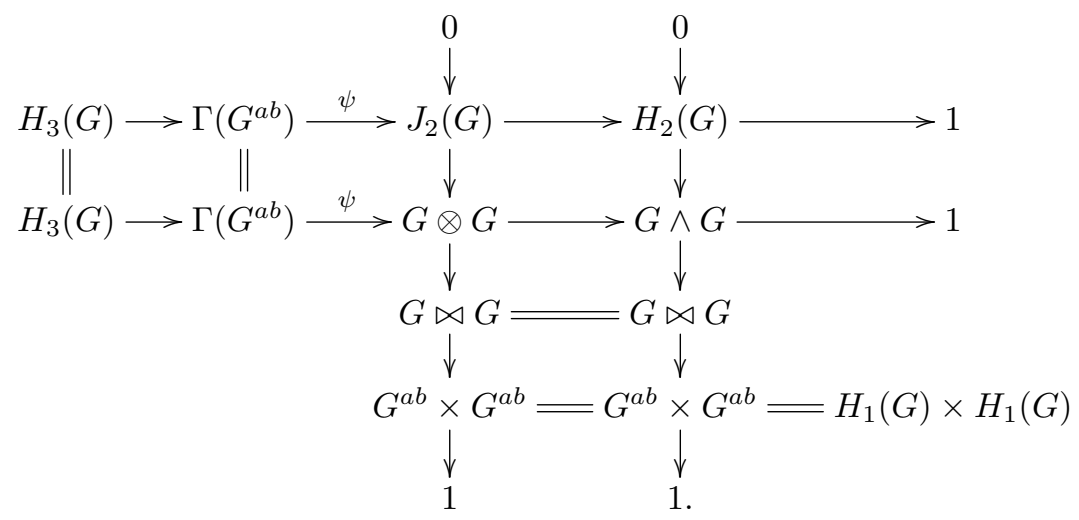




\section{Faithful linear representations}

In the paper [2] it was proved:

1) If $F_{n}$ is the free group of rank $n$, then

$$
F_{n} \otimes F_{n} \cong \mathbb{Z}^{n(n+1) / 2} \times\left(F_{n}\right)^{\prime} .
$$

2) If $N_{n, c}=F_{n} / \gamma_{c} F_{n}$ is the free nilpotent group of rank $n>1$ and class $c \geqslant 1$, then

$$
N_{n, c} \otimes N_{n, c} \cong \mathbb{Z}^{n(n+1) / 2} \times\left(N_{n, c+1}\right)^{\prime} .
$$

Proposition 6.1. Let $G$ be a free countable group. Then the exterior square $G \wedge G$ has a faithful representation into $S L_{2}(\mathbb{Z})$ and the tensor square $G \otimes G$ has a faithful representation into $G L_{2}(\mathbb{C})$.

Proof. As was proven in [7], for the free group $G$ there are isomorphisms

$$
G \wedge G \cong G^{\prime}, \quad G \otimes G \cong \Gamma\left(G^{a b}\right) \times G^{\prime} .
$$

Since $G$ is free, its commutator subgroup $G^{\prime}$ is free. Hence, by the Sanov result [12, Chapter 5] there is a faithful representation of $G^{\prime}$ into $S L_{2}(\mathbb{Z})$ and the first part of the proposition holds.

Further, $\Gamma\left(G^{a b}\right)$ is a free abelian group. Let $a_{k}, k \in I$ be its free generators. Take transcendental elements $t_{k}, k \in I$ in the field $\mathbb{C}$, which are algebraically independent over $\mathbb{Q}$. Then the matrix group

$$
T=\left\langle\left(\begin{array}{cc}
t_{k} & 0 \\
0 & t_{k}
\end{array}\right) \| k \in I\right\rangle
$$

is isomorphic to the group $\Gamma\left(G^{a b}\right)$. If $\varphi: G^{\prime} \longrightarrow G L_{2}(\mathbb{Z})$ is an embedding, then

$$
\left\langle\varphi G^{\prime}, T\right\rangle \cong \varphi G^{\prime} \times T \text {. }
$$

Hence, the group $G \otimes G$ has a faithful representation in the matrix group over the $\operatorname{ring} \mathbb{Z}\left[t_{k}^{ \pm 1}, k \in I\right]$.

If $G=F_{\infty}$ is countably generated then it has a faithful representation into $S L_{2}(\mathbb{Z})$. To prove that $\Gamma\left(F_{\infty}^{a b}\right)$ is linear we use the following property

$$
\Gamma\left(F_{\infty}^{a b}\right)=\Gamma\left(\lim F_{n}^{a b}\right)=\lim \left(\Gamma F_{n}^{a b}\right) .
$$

For the finitely generated free groups from this theorem follows

Corollary 6.2. The tensor square $F_{n} \otimes F_{n}$ has a faithful representation into $G L_{2}\left(\mathbb{Z}\left[t_{1}^{ \pm 1}, \ldots, t_{m}^{ \pm 1}\right]\right)$, where $m=\frac{n(n+1)}{2}$.

For the free nilpotent groups we can prove

Proposition 6.3. There is a faithful representation

$$
N_{n, c} \otimes N_{n, c} \longrightarrow T_{c+2}(\mathbb{C})
$$

into the group of triangular matrices $T_{c+2}(\mathbb{C})$. 
Proof. We noted that

$$
N_{n, c} \otimes N_{n, c} \cong \mathbb{Z}^{n(n+1) / 2} \times\left(N_{n, c+1}\right)^{\prime} .
$$

Hence, we have to define faithful linear representations for $\mathbb{Z}^{n(n+1) / 2}=\left\langle a_{1}, a_{2}, \ldots\right.$, $\left.a_{m}\right\rangle, m=n(n+1) / 2$ and for $\left(N_{n, c+1}\right)^{\prime}$, where $N_{n, c+1}=\left\langle x_{1}, x_{2}, \ldots, x_{n}\right\rangle$. Let

$$
\tau_{1}, \tau_{2}, \ldots, \tau_{m}, t_{i j}, i=1,2, \ldots, n, j=1,2, \ldots, c+1
$$

be complex numbers which are algebraically independent over $\mathbb{Q}$. Define the following maps

$$
a_{k} \mapsto \tau_{k} E \in T_{c+2}(\mathbb{C}), \quad k=1,2, \ldots, m,
$$

which defines a faithful representation of $\mathbb{Z}^{n(n+1) / 2}$ into $T_{c+2}(\mathbb{C})$, and

$$
x_{i} \mapsto\left(\begin{array}{ccccccc}
1 & t_{i 1} & 0 & 0 & \cdots & 0 & 0 \\
0 & 1 & t_{i 2} & 0 & \cdots & 0 & 0 \\
\vdots & \vdots & \vdots & \vdots & \ddots & \vdots & \vdots \\
0 & 0 & 0 & 0 & \cdots & 1 & t_{i c} \\
0 & 0 & 0 & 0 & \cdots & 0 & 1
\end{array}\right) \in U T_{c+2}(\mathbb{C}) \quad i=1,2, \ldots, n .
$$

As Romanovskii proved [19] the map, defined on $x_{i}$ is a faithful representation of $N_{n, c+1}$ into $U T_{c+2}(\mathbb{C})$. Hence we have a faithful representation of $\mathbb{Z}^{n(n+1) / 2} \times N_{n, c+1}$ into $T_{c+2}(\mathbb{C})$. Since $\mathbb{Z}^{n(n+1) / 2} \times\left(N_{n, c+1}\right)^{\prime}$ is a subgroup of $\mathbb{Z}^{n(n+1) / 2} \times N_{n, c+1}$, we have the needed representation.

\section{References}

[1] M. Aschenbrenner, S. Friedl and H. Wilton, 3-Manifold Groups, EMS Ser. Lect. Math., vol. 20, European Mathematical Society (EMS), Zurich, 2015.

[2] R.D. Blyth, F. Fumagalli, M. Morigi, Some structural results of the non-abelian tensor square of groups, J. Group Theory 13 (2010), 83-94.

[3] K.S. Brown, Cohomology of Groups, Grad. Texts in Math., vol. 87, SpringerVerlag, New York, 1994. x+306 pp.

[4] R. Brown, Computing homotopy types using crossed n-cubes of groups, Adams Memorial Symposium on Algebraic Topology, vol. 1 (Manchester, 1990), pp. 187-210, London Math. Soc. Lecture Note Ser., vol. 175, Cambridge Univ. Press, Cambridge, 1992.

[5] R. Brown, D.L. Johnson, E.F. Robertson, Some computations of non-abelian tensor products of groups, J. Algebra 111 (1987), 177-202.

[6] R. Brown, J.-L. Loday, Excision homotopique en basse dimension, C. R. Acad. Sci. Paris Ser. I Math. 298 (1984), 353-356.

[7] R. Brown, J.-L. Loday, Van Kampen theorems for diagrams of spaces, Topology 26 (1987), 311-335, with an appendix by M. Zisman.

[8] D.J. Collins, H. Zieschang, Combinatorial group theory and fundamental groups, Algebra, VII, pp. 1-166, 233-240, Encyclopaedia Math. Sci., vol. 58, Springer, Berlin, 1993. 
[9] G. Donadze, M. Ladra, V. Thomas, On some closure properties of the nonabelian tensor product, J. Algebra 472 (2017), 399-413.

[10] G.J. Ellis, The non-abelian tensor product of finite groups is finite, J. Algebra 111 (1987), 203-205.

[11] N.D. Gilbert, P.J. Higgins, The non-abelian tensor product of groups and related constructions, Glasg. Math. J. 31 (1989), 17-29.

[12] M.I. Kargapolov, Yu.I. Merzlyakov, Osnovy Teorii Grupp (in Russian), [Fundamentals of Group Theory], 4th edition, Fizmatlit "Nauka", Moscow, 1996.

[13] A. Kawauchi, A Survey of Knot Theory, Birkhäuser Verlag, Basel, 1996, translated and revised from the 1990 Japanese original by the author.

[14] A.S.-T. Lue, The Ganea map for nilpotent groups, J. Lond. Math. Soc. 14 (1976), 309-312.

[15] A.I. Malcev, On isomorphic matrix representations of infinite groups (in Russian), Rec. Math. [Mat. Sb.] N.S. 8 (1940), 405-422.

[16] C. Miller, The second homology group of a group; relations among commutators, Proc. Amer. Math. Soc. 3 (1952), 588-595.

[17] J. Milnor, Introduction to Algebraic K-Theory, Princeton University Press, Princeton New Jersey, 1971.

[18] I.N. Nakaoka, Non-abelian tensor products of solvable groups, J. Group Theory 3 (2000), 157-167.

[19] N.S. Romanovskii, Identity bases for certain matrix groups (in Russian), Algebra Logika 10 (1971), 401-406.

[20] M.P. Visscher, On the nilpotency class and solvability length of nonabelian tensor products of groups, Arch. Math. (Basel) 73 (1999), 161-171.

[21] B.A.F. Wehrfritz, Infinite Linear Groups, Berlin, 1973.

[22] J.H.C. Whitehead, On adding relations to homotopy groups, Ann. of Math. 42 (1941), 409-428.

Valeriy G. Bardakov bardakov@math.nsc.ru

Sobolev Institute of Mathematics, Acad. Koptyug Avenue, 4, Novosibirsk State University, Pirogova Street 1, Novosibirsk 630090, Russia

Novosibirsk State Agrarian University, Dobrolyubova Street, 160, Novosibirsk 630039, Russia

Andrei V. Lavrenov A.V.Lavrenov@gmail.com

Saint-Petersburg State University, University Embankment, 7-9, Saint-Petersburg 199034, Russia

Mikhail V. Neshchadim neshch@math.nsc.ru

Sobolev Institute of Mathematics, Acad. Koptyug Avenue, 4, Novosibirsk State University, Pirogova Street 1, Novosibirsk 630090, Russia 